\title{
Individual-based Information Dissemination in Multilayer Epidemic Modeling
}

\author{
F.D. Sahneh ${ }^{1}$, F.N. Chowdhury ${ }^{2}$, G. Brase ${ }^{3}$, C.M. Scoglio ${ }^{1 *}$ \\ ${ }^{1} \mathrm{~K}$-State Epicenter, Department of Electrical and Computer Engineering \\ Kansas State University, Manhattan, KS 66506, USA \\ ${ }^{2}$ Directorate for Social, Behavioral \& Economic Sciences, National Science Foundation \\ Arlington, VA 22230, USA \\ ${ }^{3}$ Department of Psychological Sciences, Kansas State University \\ Manhattan, KS 66506, USA
}

\begin{abstract}
In epidemic modeling, the Susceptible-Alert-Infected-Susceptible (SAIS) model extends the SIS (Susceptible-Infected-Susceptible) model. In the SAIS model, "alert" individuals observe the health status of neighbors in their contact network, and as a result, they may adopt a set of cautious behaviors to reduce their infection rate. This alertness, when incorporated in the mathematical model, increases the range of effective/relative infection rates for which initial infections die out. Built upon the SAIS model, this work investigates how information dissemination further increases this range. Information dissemination is realized through an additional network (e.g., an online social network) sharing the contact network nodes (individuals) with different links. These "information links" provide the health status of one individual to all the individuals she is connected to in the information dissemination network. We propose an optimal information dissemination strategy with an index in quadratic form relative to the information dissemination network adjacency matrix and the dominant eigenvector of the contact network. Numerical tools to exactly solve steady state infection probabilities and influential thresholds are developed, providing an evaluative baseline for our information dissemination strategy. We show that monitoring the health status of a small but "central" subgroup of individuals and circulating their incidence information optimally enhances the resilience of the society against infectious diseases. Extensive numerical simulations on a survey-based contact network for a rural community in Kansas support these findings.
\end{abstract}

Keywords and phrases: Epidemic model, infectious disease, SAIS, information dissemination

Mathematics Subject Classification: $92 \mathrm{~B} 99$

\section{Introduction}

Visitors to Asia may notice that a small, but noticeable, portion of the population there is in the habit of wearing hospital masks when out in public. These are not people who are themselves harboring contagious

${ }^{*}$ Corresponding author. E-mail: caterina@ksu.edu 
disease (any more than anyone is), nor are they all just returning from a particularly hazardous location. They are, quite simply, in a heightened state of alertness regarding airborne infectious diseases and showing a visible aspect of this alert state. It is not the only form of behavioral change that can occur because someone is in an alert state (others include increased hand washing, decreased contact with other people, and receiving immunization shots), but it is an immediately observable one. It leads to a key question regarding the role of individual behaviors in modeling epidemics: How do peoples' behaviors influence the emergence, progression, and eventual resolution of epidemics in a population? Studying individual human responses to a new emerging pathogen (e.g. sudden and zoonotic in origin) is vital for social health. To study the impact of individual behavioral responses to epidemics taking into account the structure of the contact network and, more generally, of the social networks connecting individuals, simple but effective models needs to be developed.

Mathematical and computational epidemiology is now contributing to the study of human behaviors during the course of epidemic spreading [4]. The challenges in this topic concern not only how to model human reactions to the presence of epidemics, but also how these reactions affect the spread of the disease itself [3]. In a general view, human preventive responses to an epidemic spread can be categorized into the following three types: 1) change in the individual's state [17], 2) change in epidemic model parameters [24] and 3) change in the contact topology [16,25]. A comprehensive review of the literature on interaction of the epidemic spreading and human behavior is available in [5]. Poletti et al. developed a population-based model where susceptible individuals could choose between two behaviors in response to presence of infection [15]. Funk et al. showed that awareness of individuals about the presence of a disease can help reducing the size of the epidemic outbreak [6]. In their paper, awareness and disease have interconnected dynamics. Perra et al. [14] considered the case where individuals go to a 'feared' state when they sense infection. These population-based models are suitable for a society of well-mixed individuals, neglecting heterogeneity of social interactions.

The characteristics of classic epidemic models have been studied for a long time and are well-established. In susceptible-infected-susceptible (SIS) and susceptible-infected-removed (SIR) epidemic models, the ratio between the infection rate $\beta$ and the recovery rate $\delta$, called here effective infection rate $\tau=\beta / \delta$ characterizes the aggressiveness of infectious diseases.

In our previous paper [18], we derived thresholds in the case of spontaneous behavioral responses, and assessed the capability of human behavioral responses to influence epidemic spreading. We established two critical values for the effective infection rate qualitatively dividing the dynamical response of the models into three regions. For infectious diseases with effective infection rate below the first critical value, initial infections do not spread and die out quickly. When effective infection rates are beyond this critical value, however, initial infections start growing. In this scenario, a second threshold becomes evident. When initial infection gets the chance to grow, people in the population can react to the infection by adopting preventive behaviors after receiving the information of the infection spreading. If this behavioral reaction is strong enough, it can effectively contain the infection. This second threshold depends on the behavioral response for which a key element is information about infection incidences, enabling awareness and potential corrective behaviors.

In Figure 1, the structure of the contact network and the online social links among individuals are shown. We provide new insights into the efficacy of individuals extracting and disseminating information from their physical neighbors and on-line social contacts.

Importantly, the information that each person (represented by single node in network models) is able to receive can be described as coming from two fundamentally different sources. One source of information is direct experience of people from their immediate contact (adjacent nodes), which carries an inherent concomitant risk of infection. The other source of information is from electronically-mediated information dissemination networks (i.e., on-line social networks such as Facebook@ and Twitter@, mass communication sources such as radio and television, and also personal communication through email and phone). This second source of information is not associated with a change in actual risk of infection, but still carries information about disease states of other people (nodes). On-line social networks also 


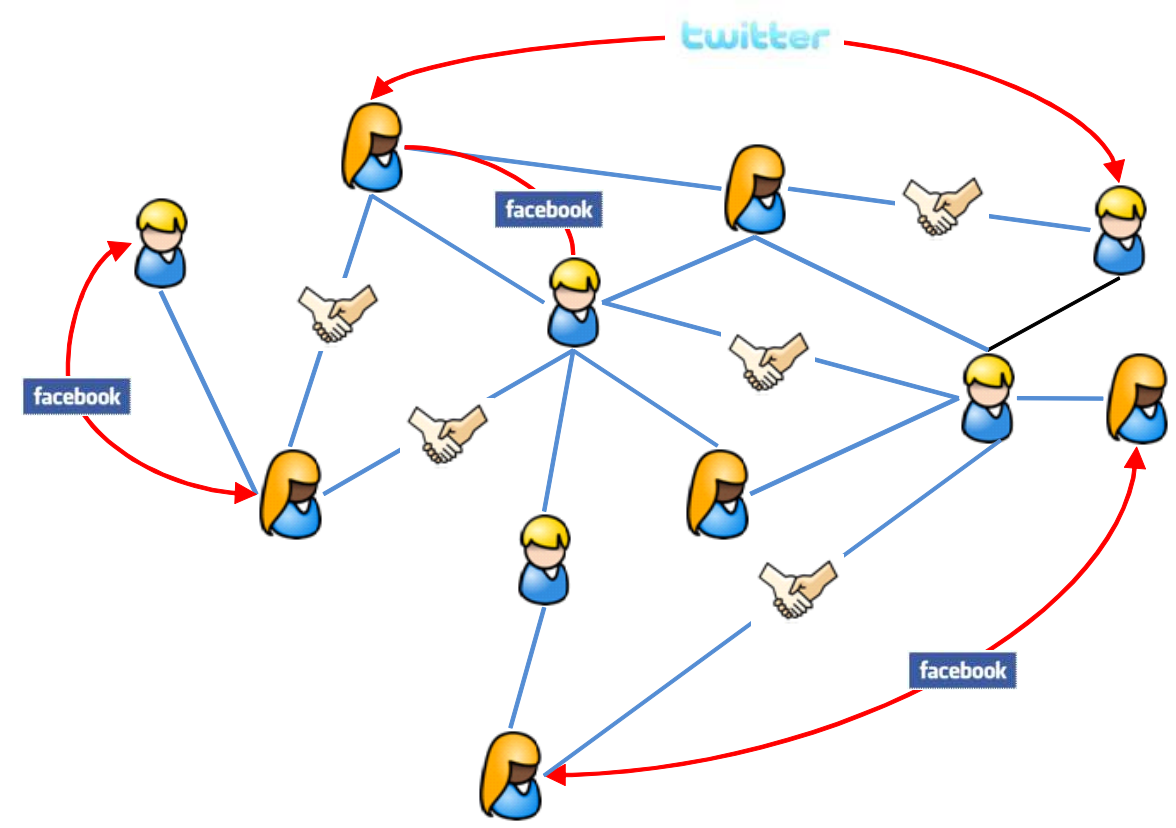

FiguRe 1. Two social network layers: one layer represents physical contact (blue links) and the other layer represents information exchange through online social networks (red links)

transmit information across vastly greater geospatial distances, easily carrying disease information faster than the actual disease transmission.

The development of the two thresholds in [19] is mathematically elegant, yet the impact of the information dissemination network structure on these thresholds is not fully understood. In this paper, we provide rigorous results describing the impact of information dissemination networks and demonstrate how these networks can be designed to incentivize preventive behaviors.

The contributions of this paper can be summarized as follows. We utilize the information dissemination index defined in [19] for the susceptible-alert-infected-susceptible model with information dissemination (SAIS-ID), to develop further results and explain the impact of information dissemination on the epidemic spread or die-out. In this context, we show 1) how maximizing the information dissemination index can expand the region for effective infection rate where the epidemic eventually dies out, 2) how to select the optimal structure of the information dissemination network, and 3) how to quantify the gain from this optimal structure. Aside from application and interpretation of theoretical results of [19], we also derive numerical tools to exactly find final infection probabilities and critical parameters, hence complementing our earlier approximate analytical results.

\section{Models}

The spreading of infectious diseases has been mathematically modeled and studied for a long time. Among the most widely used and important epidemic models is the SIS (Susceptible-Infected-Susceptible) model, where each individual in the population can either be susceptible or infected. In this model (see Figure 2 ), an infected person (node) can infect its neighbors in the contact network with an infection rate $\beta$, and the infection is cured with a recovering rate $\delta$. However, once cured and healthy, the person is again prone to the infection. According to this model applied to a general contact network, the probability of being infected is shown to follow the solution of a set of differential equations. Despite being very simple, the SIS model has been a good mathematical tool for studying the spreading of infectious diseases such 


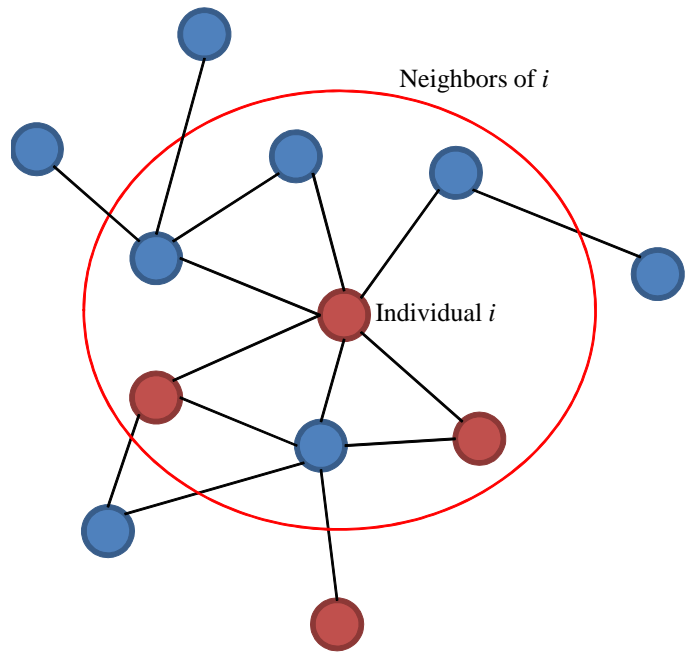

Figure 2. Epidemic spreading schematics in SIS model. Node $i$ is infected (red) due to contact with its infected neighbors, black links show contact between individuals.

as the common cold. Analyzing the SIS model, an interesting behavior is detected. If $\beta / \delta$ is smaller than a certain threshold $\tau_{c}$, an initial small number of infections will die out and the number of infected individuals will go to zero. Conversely, if $\beta / \delta$ is greater than the same threshold, an initial small number of infections will spread, and the number of infected individuals will increase converging toward a positive non-zero steady state value. This threshold value $\left(\tau_{c}\right)$ is usually refered to epidemic threshold in the mathematical modeling community.

The term epidemic threshold, unfortunately, has several other definitions in different scientific communities. In this paper, we refer to the critical values of infection rate as 'die-out threshold' and 'no-spreading threshold,' to be defined precisely in Section 3. These critical values serve a role similar to that of the basic reproduction number $R_{0}$, which has a more universally accepted definition as the number of secondary infections from a single initial infection in a susceptible population. If $R_{0}<1$ the initial infections die out, and if $R_{0}>1$, initial infections grow [9].

\subsection{The SAIS model}

In [17], Sahneh and Scoglio proposed a preventive behavioral response model coupled with the SIS model to account for preventive human behaviors. Specifically, upon observation of infection, individuals adopt more cautious behaviors. Those individual with alert behaviors have a lower infection rate. The alert individuals are represented by a new compartment, denoted by 'alert.' In this article, we refer to this model as Susceptible-Alert-Infected-Susceptible (SAIS). Both susceptible and alert individuals can potentially be infected; however, the infection rate for the alert individuals is lower. In other words, in the SAIS model, the adoption of preventive behaviors is effectively modeled as a reduction in the infection rate parameter. Even though classic approaches suggested from public health officials have focused primarily on recommending preventive behavioral changes to infected individuals, recent studies [2] show that alert behaviors need to be extended to susceptible individuals to be effective. Furthermore, this behavior can be thought of as multifaceted and encompassing an array of adopted hygienic behaviors. Therefore, it would be more realistic to consider different levels of reduction in the infection rate. However, since the goal of this paper is to study the influence of information dissemination networks, we only consider a single reduction of the infection rate in order to avoid unnecessary complications of the model.

In our work, the contact network is represented by a graph with $N$ nodes (individuals) and $L$ links (contacts) and its adjacency matrix $A$ of size $(N \times N)$. Each individual is allowed to be in one of three 
states 'susceptible,' 'infected,' and 'alert.' Figure 3 shows the transition diagram in this model for each individual. A susceptible individual becomes infected by the infection rate $\beta$ times the number of her infected neighbors in $A$. An infected individual recovers back to the susceptible state by the recovery rate $\delta$. An individual can observe the states of her neighbors. A susceptible individual might go to the alert state if surrounded by infected individuals, as shown in Figure 4. Specifically, a susceptible node becomes alert with the alerting rate $\kappa$ times the number of infected neighbors. The alert rate $\kappa$ parameter reflects three aspects of the alerting process: 1) the chance of observing an infected neighbor, 2) the probability of making the decision to become alert, and 3) the rate at which an alert behavior is adopted. In other words $\kappa$ includes the imperfect availability of observation.

An alert individual can get infected in a process similar to a susceptible individual but with a smaller infection rate $\beta_{a}, 0 \leq \beta_{a}<\beta$, due to preventive behaviors. This assumption is supported by the literature (e.g., $[1,13])$. Furthermore, we assume that transition from the alert back to the susceptible state is much slower than other transitions in the system. This assumption is reasonable in some scenarios, and is supported by both neuroscience and behavioral sciences findings, e.g., sympathetic nervous system activation is generally faster than its return to resting state [21]; decay back to resting state is slower than initial activation in neural network models [10]; the time course of distraction during focused attention [12]. Hence, in our modeling setup, we assume that there is not enough time for an alert individual to go back directly to the susceptible state. SAIS approach separates disease model from behavioral response model with a feedback structure.

Epidemic parameters $\beta, \delta, \beta_{a}$, and $\kappa$ are not directly measurable. Estimation techniques like Monte Carlo Markov Chain (MCMC) [7] are common approaches to estimate epidemic parameters from incidence data. Estimation of epidemic parameters is beyond the scope of this paper. We conduct a parametric analysis of the spreading dynamics and our final results prove to be independent of epidemic parameters exact values.

The overall dynamics of SAIS stochastic transitions follow a coupled Markov process. For a network with arbitrary structure, this model becomes mathematically intractable due to exponential explosion of its Markov state space size [20]. To overcome this issue, applying closure techniques (see, [23]) results in approximate models with much smaller state space size, however at the expense of accuracy. Specifically, a first order mean-field type approximation, the GEMF framework [20] suggests the following differential of size $2 N$ :

$$
\begin{aligned}
& \dot{p}_{i}=\beta\left(1-p_{i}-q_{i}\right) \sum_{j=1}^{N} a_{i j} p_{j}+\beta_{a} q_{i} \sum_{j=1}^{N} a_{i j} p_{j}-\delta p_{i} \\
& \dot{q}_{i}=\kappa\left(1-p_{i}-q_{i}\right) \sum_{j=1}^{N} a_{i j} p_{j}-\beta_{a} q_{i} \sum_{j=1}^{N} a_{i j} p_{j}
\end{aligned}
$$

for $i \in\{1, \ldots, N\}[17]$.

In these equations, $p_{i}$ is the probability of node $i$ being infected, $q_{i}$ is the probability of node $i$ being alert, and $\left(1-p_{i}-q_{i}\right)$ is the probability of node $i$ being susceptible; $a_{i j}$ is the $(i, j)$ element of the adjacency matrix for the contact network (or contact graph) $A$. If individual $i$ and individual $j$ are in physical contact $a_{i j}=a_{j i}=1$, if they are not in contact $a_{i j}=a_{j i}=0$. We use here the symbol $A$ to represent both the contact network and its adjacency matrix, and should not be confused with the alert state A.

\subsection{SAIS Model with Information Dissemination}

We now turn to model how information dissemination via online social networks can impact the characteristics of the SAIS model. We assume that the information dissemination is realized through an additional network among individuals, which has the same $N$ nodes but different links with respect to the contact network. Each link in the information dissemination network is a directed link which provides the health 


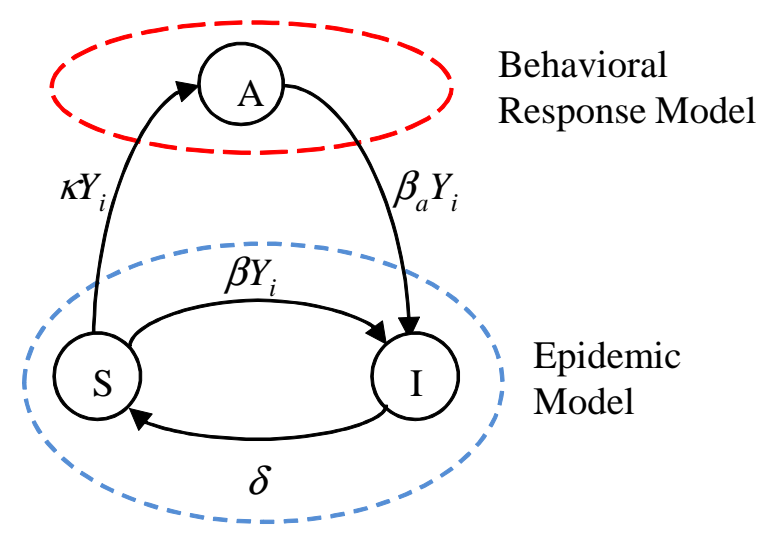

Figure 3. Transition diagram for the states of individual (node) $i$ in the SAIS model, where $Y_{i}$ is the number of infected neighbors of individual $i$

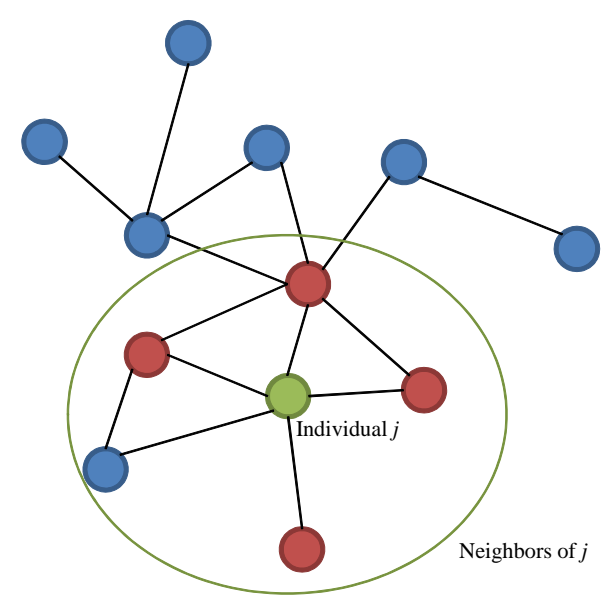

FIGURE 4. Epidemic spreading schematics in SAIS model. Individual (node) $j$ is alert (green) due to the observation of its infected contact neighbors.

status of the source individual to the sink individual. We represent the information dissemination network and its adjacency matrix with $B$. The $(i, j)$ element of the adjacency matrix of the information dissemination network (or information dissemination graph) $B$ is $b_{i j}$. If individual $i$ receives the health status of individual $j$ in the dissemination network $b_{i j}=1$, otherwise $b_{i j}=0$. The adjacency matrix $B$ of size $(N \times N)$ in general is not symmetric since the dissemination network is a directed graph. From now on, we refer to the SAIS model with information dissemination network as SAIS-ID model.

To describe the process of transition to the alert state due to information spread, we introduce $\mu$ as the rate at which susceptible individuals become alert when they learn that their neighbors in the information dissemination network are infected.

When considering the information dissemination network (Figure 6), the transition rate of a susceptible individual to the alert state is increased by an additional component expressed by the product of the parameter $\mu$ times the number of infected neighbors $Z_{i}$ observed through the information dissemination network $B$. 


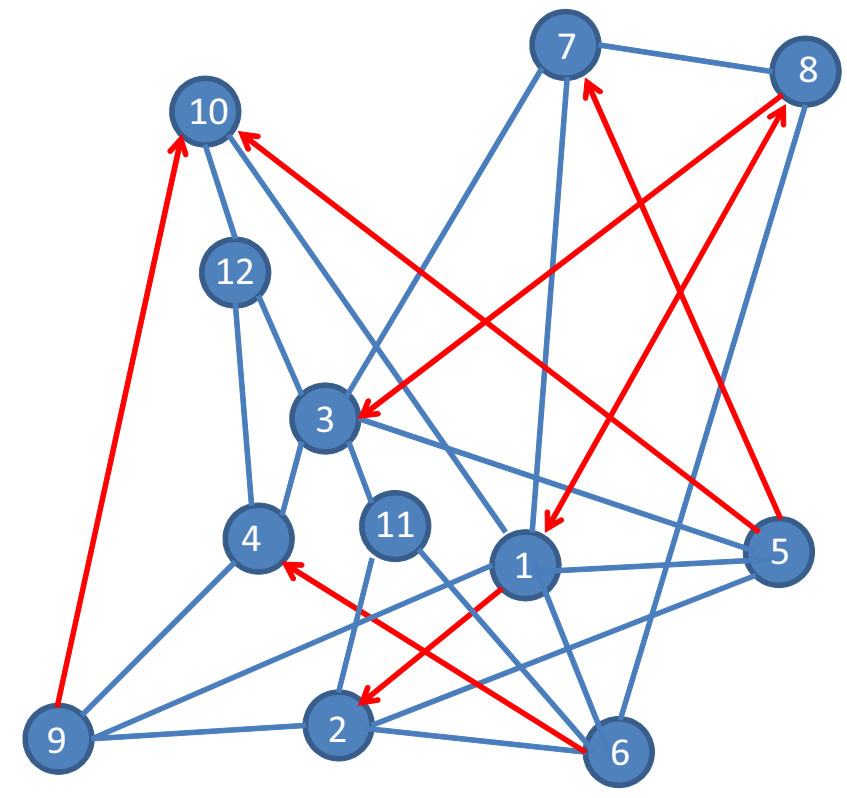

FiguRE 5. Multilayer network topology of SAIS-ID model. Individuals receive information and potentially infection from individuals through blue links, while only information is sent through red links.

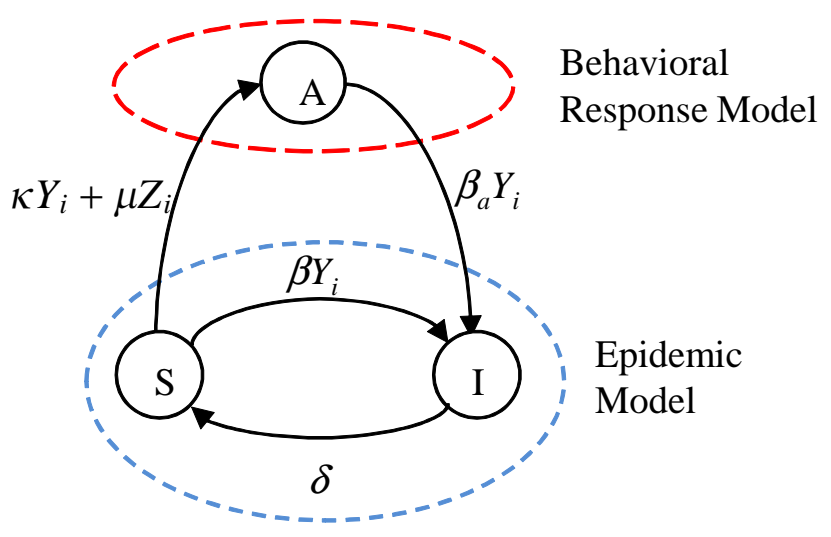

Figure 6. Transition diagram for the states of individual (node) $i$ in the SAIS-ID model, where $Y_{i}$ is the number of infected neighbors of individual $i$, and $Z_{i}$ is the number of neighbors of individual $i$ whose infection is obtained through the information network.

Using the GEMF framework [20] for developing mean-field epidemic models in multi-layer networks, we obtain the following $2 N$ differential equations:

$$
\begin{aligned}
& \dot{p}_{i}=\beta\left(1-p_{i}-q_{i}\right) \sum_{j=1}^{N} a_{i j} p_{j}+\beta_{a} q_{i} \sum_{j=1}^{N} a_{i j} p_{j}-\delta p_{i}, \\
& \dot{q}_{i}=\left(1-p_{i}-q_{i}\right)\left\{\kappa \sum_{j=1}^{N} a_{i j} p_{j}+\mu \sum_{j=1}^{N} b_{i j} p_{j}\right\}-\beta_{a} q_{i} \sum_{j=1}^{N} a_{i j} p_{j},
\end{aligned}
$$




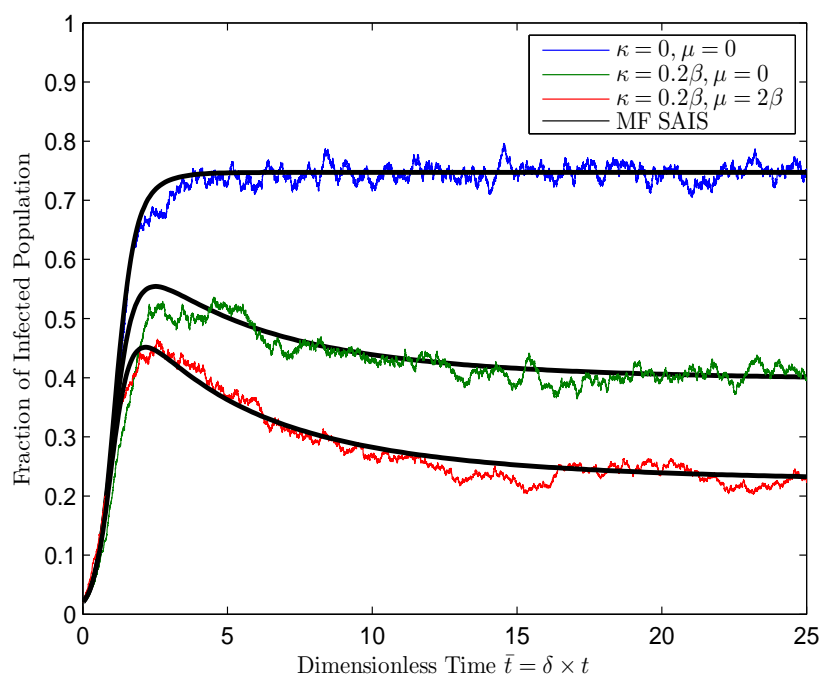

Figure 7. Comparison between mean-field model and Gillespie simulation for a dense contact network. Vertical axis denotes the fraction of infected population for different alertness rates. In this simulation, number of nodes is $N=1000$. The contact graph is a geometric random graph with critical connection radius $r=\sqrt{2 \log (N) / N}$, where average node degree is $\bar{k}=12.85$ and its average local clustering coefficient is $\bar{C}=0.5978$. The information dissemination network is a Barabasi-Albert scale free network with powerlaw exponent $\gamma=3$. The effective infection rate is $\beta / \delta=5 / \lambda_{1}(A)$.

for $i \in\{1, \ldots, N\}$ [19]. The SAIS-ID model (2.3-2.4) is similar to (2.1-2.2) with the main difference of a higher rate for alertness process due to information dissemination.

The mean-field type approximation introduces some error to the infection probabilities of nodes [8]. This error is small for a wide range of network topologies and epidemic related parameters. However, for sparse structured graphs and networks with heavy tail distributions the error is larger. In particular, for epidemic values close to critical values, the error becomes significant. As an illustration, we have numerically simulated the stochastic SAIS-ID model versus the mean-field approximate SAIS-ID model. In this example, the physical contact network is a geometric random network and the information dissemination network is generated according to Barabasi-Albert scale free model. In particular, we compare two cases of random geometric network with $r=\sqrt{2 \log (N) / N}$ and another one with $r=\sqrt{1.1 \log (N) / \pi N}$. Simulations in Figure 7 show very good agreement between stochastic simulations and mean-field model when the contact network is dense while there is a large error for the case of sparse geometric graph as shown in Figure 8. We have selected the recovery rate is $\delta=1$ to make simulation time dimensionless.

\section{Results}

In this section, we derive threshold values as well as optimal topology of information dissemination network for the SAIS-ID model presented in the previous section. In addition, we develop numerical tools for computational determination of threshold values and steady-state infection probabilities.

\subsection{SAIS-ID Model Analysis}

Using the SAIS-ID model presented in [19], two interesting thresholds can be computed, one of which can be influenced by individual behaviors and information dissemination, with potential benefits for public health purposes. 


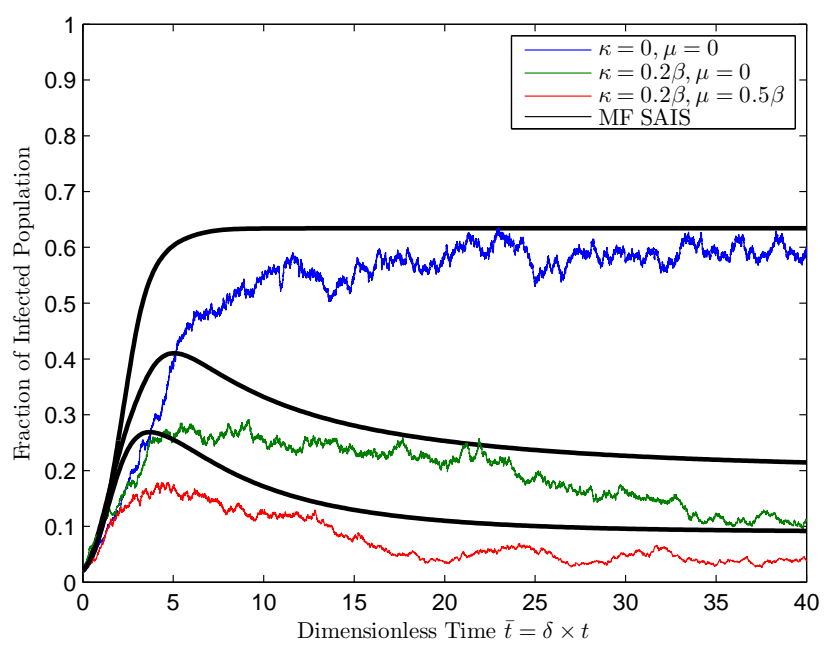

FiguRE 8. Comparison between mean-field model and Gillespie simulation for a sparse contact network. All the settings are similar to Figure 7 except the threshold connection radius value in geometric random graph model is $r=\sqrt{1.1 \log (N) / \pi N}$ to obtain a sparse contact network with average node degree $\bar{k}=7.168$ and average local clustering coefficient $\bar{C}=0.5843$.

As discussed earlier, each SIS modeled infectious disease is characterized by its effective infection rate $\tau$ given by the ratio between the infection rate $\beta$ and the recovery rate $\delta$,

$$
\tau=\frac{\beta}{\delta}
$$

These parameters are modified, though, when someone is in an alert state. The alert effective infection rate $\tau_{a} \triangleq \frac{\beta_{a}}{\delta}$ is defined as the ratio between the alert infection rate $\beta_{a}$ and the recovery rate $\delta$, where $\beta_{a}<\beta$, due to the preventive behaviors of alert individuals.

In [18], we have shown SAIS model (2.1-2.2) exhibits three types of behavior: (a) quick die-out, (b) slow die-out, and (c) epidemic spreading, as shown in Figure 9. In the quick die-out region, the epidemic dies out exponentially, whereas in the slow die-out region, the epidemic spreads at the beginning, but after some time it dies out as the result of increased alertness in the network. For the last case, the infected population fraction never goes to zero and stabilizes at a constant value above zero.

\subsubsection{No-spreading Threshold}

The no-spreading threshold for the effective infection rate $\tau_{c 1}$ is related to the level or amount of physical contact in the population in the SIS model. A specific pathogen cannot spread in a population if its specific effective infection rate is lower than the no-spreading threshold. The level of contact must be sufficiently high for a pathogen with a given effective infection rate to spread in the population. Stronger and more frequent contacts in a population provide more opportunities for pathogen spreading, making the no-spreading threshold inversely related to the contact level.

For example, if everybody is in contact with $d$ individuals and the contact network is a regular graph with constant node degree, then the threshold is inversely proportional to $d$ [9].

When the contact network is a general graph, the structure of the graph can be mathematically represented by the adjacency matrix $A$. In this case the level of contact among individuals is represented by the largest eigenvalue of the adjacency matrix, $\lambda_{1}$. Summarizing, the no-spreading threshold only depends on the contact network and is inversely proportional to the contact level.

$$
\tau_{c 1}^{\text {regular }}=\frac{1}{d}, \quad \tau_{c 1}^{\text {general }}=\frac{1}{\lambda_{1}}
$$




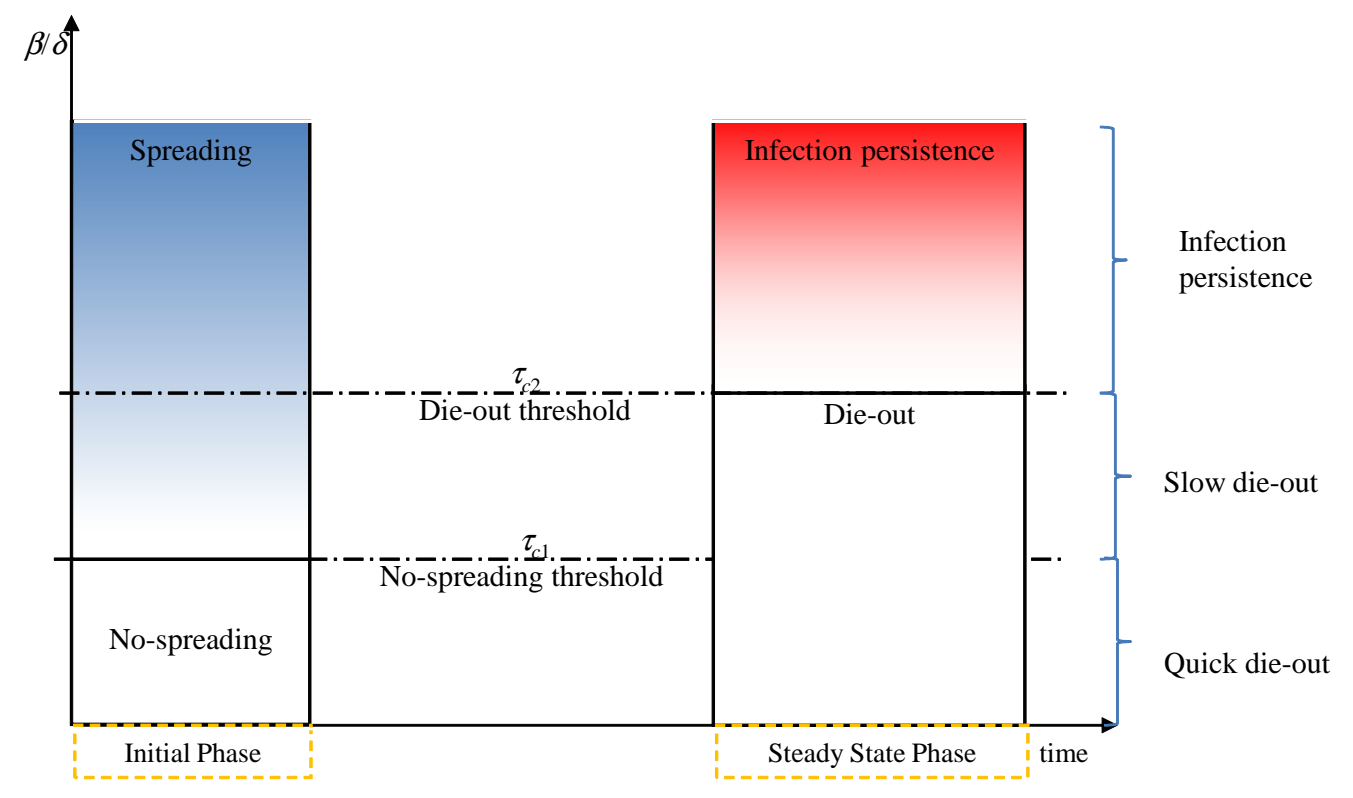

Figure 9. No-spreading threshold and die-out threshold qualitatively divide the total range of effective infection rate into three regions: when the effective infection rate $\beta / \delta$ is smaller than the first threshold, the infection does not spread and dies out quickly; between the two thresholds the infection initially spreads, but later, due to the alertness effect, it dies out slowly; finally, when the effective infection rate $\beta / \delta$ is greater than the second threshold, the infection persists in the population.

It is important to understand that the meaning and interpretation of "contact level" varies with the specific disease considered and its means of transmission. In the simple scenario of an airborne infection, contact level can represent the distance, intensity, and the frequency of face-to-face encounters.

\subsubsection{Die-out threshold}

The die-out threshold $\tau_{c 2}$ is related to both the contact level and the alertness process parameters of the SAIS model [19]. When considering an alertness process, an initial increase of the number of infected individuals triggers the spreading of the alertness process, which in turn can contain and, in some cases, fully suppress the epidemic.

For the SAIS model without information dissemination $(\mu=0)$, the die-out threshold is found as follows [18]:

$$
\tau_{c 2}(0)=\tau_{c 1}+\frac{\kappa}{\beta_{a}}\left(\tau_{c 1}-\tau_{a}\right)
$$

The above equations demand the condition $\tau_{a}<\tau_{c 1}$ in order to get $\tau_{c 2}>\tau_{c 1}$. This condition has interesting interpretations: the preventive behavior of alert individuals should be effective enough to suppress the infection if all individuals adopt the preventive behavior. Since the alert effective infection rate $\left(\tau_{a}\right)$ is smaller than the no-spreading threshold $\left(\tau_{c 1}\right)$, the die-out threshold $\tau_{c 2}$ is larger than the no-spreading threshold and the three regions are correctly defined.

Similar to the SAIS model (2.1-2.2), initial-time dynamics of infection spreading is not influenced by behavioral factors for the SAIS-ID model. Therefore, addition of information dissemination network does not change the no-spreading threshold. However, the information dissemination network increases the die-out threshold by promoting alert behaviors. The die-out threshold $\tau_{c_{2}}$ for SAIS-ID model $(2.3,2.4)$ is the solution to the following nonlinear eigenvalue problem [19] 


$$
\tau_{c_{2}} \sum_{j=1}^{N} a_{i j} w_{j}-\left(\tau_{c_{2}}-\frac{\beta_{a}}{\delta}\right) f_{i}(\boldsymbol{w}, \mu) \sum_{j=1}^{N} a_{i j} w_{j}-w_{i}=0,
$$

where $f_{i}(\boldsymbol{w}, \mu)$ is defined as

$$
f_{i}(\boldsymbol{w}, \mu) \triangleq \frac{\frac{\kappa}{\beta_{a}} \sum_{j=1}^{N} a_{i j} w_{j}+\mu \sum_{j=1}^{N} b_{i j} w_{j}}{\left(1+\frac{\kappa}{\beta_{a}}\right) \sum_{j=1}^{N} a_{i j} w_{j}+\mu \sum_{j=1}^{N} b_{i j} w_{j}} .
$$

Unfortunately, this nonlinear eigenvalue problem does not have an exact explicit solution. Using an eigenvalue perturbation method, we found a function with quadratic form provides an explicit approximate solution to the nonlinear eigenvalue problem [19]. We refer to this function as information dissemination index.

Definition 1: For a generic contact network $A$ and a generic information dissemination network $B$, we define the information dissemination index $\phi_{A}(B)$ as:

$$
\phi_{A}(B)=\frac{v_{1}^{T} B v_{1}}{\lambda_{1}}
$$

where $v_{1}$ is the normalized dominant eigenvector of the adjacency matrix $A$ corresponding to its largest eigenvalue $\lambda_{1}$.

The information dissemination index $\phi_{A}(B)$ corresponds to the interrelation of the physical contact network and information dissemination network. This index quantifies information transmission between nodes depending on their spectral centrality. The more spectrally central nodes are connected through information dissemination network, the larger this index. Interestingly, when the information network is the same as the contact network, i.e., $B=A$, the information dissemination index is equal to one, i.e., $\phi_{A}(B)=\phi_{A}(A)=1$. When the information network is a directed subgraph of the contact graph, then $\phi_{A}(B)<1$. The information dissemination index $\phi_{A}(B)$ is an important quantity to reflect the impact of the information dissemination network

For the SAIS-ID model, the die-out threshold (3.2) is modified as [19]:

$$
\tau_{c 2} \simeq \tau_{c 1}+\left(\frac{\kappa}{\beta_{a}}+\phi_{A}(B) \frac{\mu}{\beta_{a}}\right)\left(\tau_{c 1}-\tau_{a}\right) .
$$

Similar to contact alerting rate $(\kappa)$, the information dissemination alerting rate $(\mu)$ increases the dieout threshold. However, this increase is (almost) proportional to the information dissemination index $\phi_{A}(B)$ introduced in Definition 1. In other words, the information dissemination index determines the efficacy of the information dissemination network $B$ with respect to the contact network $A$.

Information dissemination index $\phi_{A}(B)$ defined in (3.5) has an interesting interpretation. We rewrite $\phi_{A}(B)$ as

$$
\phi_{A}(B)=\frac{v_{1}^{T} B v_{1}}{\lambda_{1}}=\frac{1}{\lambda_{1}} \sum_{(i, j) \in E_{B}} v_{1, i} v_{1, j},
$$

where $E_{B}$ is the set of edges of $B$, and $v_{1, i}$ is the $i$-th element of eigenvector $v_{1}$. If we interpret $v_{1, i} v_{1, j}$ as a measure of how central two connected nodes are, the information dissemination index $\phi_{A}(B)$ interprets as the summation of these edge centralities.

\subsection{Numerical Tools}

The steady state infection probability of each node from (2.3-2.4) is an indicator of its expected long-term infection state. The average of these infection probabilities is an estimate of the final infection size in SAIS-ID model. Interestingly, the final infection fraction as a function of effective infection rate $\tau$ explains the die-out threshold phenomenon: for effective infection rates smaller than the die-out threshold, the 
final infection fraction is zero, while for effective infection rates greater than the die-out threshold, final infection fraction is positive and increasing monotonically with $\tau$.

The steady state infection probabilities can be obtained by running the ODE equations of (2.3-2.4) for a sufficiently long time. However, this method is time-consuming because continuous-time simulation of the differential equations demands incremental time steps. In this paper, we propose a discrete recursive formula that solves the equilibrium probabilities

$$
\begin{aligned}
\frac{p_{i}^{*}}{1-p_{i}^{*}-q_{i}^{*}} & =\tau \sum_{j=1}^{N} a_{i j} p_{j}^{*}+\tau_{a} \frac{q_{i}^{*}}{1-p_{i}^{*}-q_{i}^{*}} \sum_{j=1}^{N} a_{i j} p_{j}^{*}, \\
\frac{q_{i}^{*}}{1-p_{i}^{*}-q_{i}^{*}} & =\frac{\mu}{\beta_{a}} \sum_{j=1}^{N} b_{i j} p_{j}^{*}+\frac{\kappa}{\beta_{a}},
\end{aligned}
$$

obtained from (2.3-2.4). Defining auxiliary states $y_{i}$ and $z_{i}$ as,

$$
\begin{aligned}
y_{i}^{*} & =\frac{p_{i}^{*}}{1-p_{i}^{*}-q_{i}^{*}}, \\
z_{i}^{*} & =\frac{q_{i}^{*}}{1-p_{i}^{*}-q_{i}^{*}},
\end{aligned}
$$

the equilibrium equation (3.7-3.8) can be re-expressed by

$$
\begin{aligned}
& y_{i}(k+1)=\tau \sum_{j=1}^{N} a_{i j} \frac{y_{j}(k)}{1+y_{j}(k)+z_{j}(k)}+\tau_{a} z_{i}(k) \sum_{j=1}^{N} a_{i j} \frac{y_{j}(k)}{1+y_{j}(k)+z_{j}(k)}, \\
& z_{i}(k+1)=\frac{\mu}{\beta_{a}} \sum_{j=1}^{N} b_{i j} \frac{y_{j}(k)}{1+y_{j}(k)+z_{j}(k)}+\frac{\kappa}{\beta_{a}} .
\end{aligned}
$$

The above update law has the form of contraction mapping. According to contraction mapping theorem, above fixed point iteration converges exponentially as $k \rightarrow \infty$, solving steady-state infection and alertness probabilities as

$$
\begin{aligned}
& \frac{y_{i}(k)}{1+y_{i}(k)+z_{i}(k)} \rightarrow p_{i}^{*}, \\
& \frac{z_{i}(k)}{1+y_{i}(k)+z_{i}(k)} \rightarrow q_{i}^{*} .
\end{aligned}
$$

The main benefit of this method is finding the steady-state probabilities fairly accurately in finite time steps. In Figure 11, we apply this method to show how information dissemination increases the die-out threshold, plotting final infected population fraction $\bar{p}_{s s}=\sum_{i=1}^{N} p_{i}^{*}$ as a function on effective infection rate $\tau$.

Another major contribution of this work is a numerical method to solve the nonlinear eigenvalue problem (3.3) for finding die-out threshold. A numerical method to find the die-out threshold is crucial to evaluate the performance of our proposed information dissemination index (3.5). The die-out threshold equation (3.3) can be expressed in the collective form

$$
\tau_{c_{2}} A \boldsymbol{w}-\left(\tau_{c_{2}}-\frac{\beta_{a}}{\delta}\right) F(\boldsymbol{w}) A \boldsymbol{w}-\boldsymbol{w}=0
$$

where $\boldsymbol{w}=\left\{w_{1}, \ldots, w_{N}\right\}$ and $F(\boldsymbol{w})=\operatorname{diag}\left\{f_{i}(\mathbf{w}, \mu)\right\}$. Hence, the die-out threshold is the solution to nonlinear Perron-Frobenius equation

$$
\boldsymbol{w}=\tau_{c_{2}}\left(I-\tau_{a} F(\boldsymbol{w}) A\right)^{-1}(I+F(\boldsymbol{w})) A \boldsymbol{w} .
$$


The term $\left(I-\tau_{a} F(\boldsymbol{w}) A\right)$ is guaranteed to be invertible because $\tau_{a}<\frac{1}{\lambda_{1}(A)}$ by definition and $F(\boldsymbol{w})$ is a diagonal matrix with entries less than 1 . This equation can be effectively solved via iterative law

$$
\boldsymbol{w}(k+1)=\frac{\digamma(\boldsymbol{w}(k))}{\|\digamma(\boldsymbol{w}(k))\|_{2}},
$$

where $\digamma(\boldsymbol{w})$ is defined as

$$
\digamma(\boldsymbol{w})=\left(I-\tau_{a} F(\boldsymbol{w}) A\right)^{-1}(I+F(\boldsymbol{w})) A \boldsymbol{w} .
$$

The update law (3.17) is an iteration of nonlinear maps for nonlinear Perron-Frobenius (see [11]) problem $\boldsymbol{w}=\digamma(\boldsymbol{w})$ and converges exponentially, solving $\tau_{c 2}$ as $\frac{1}{\boldsymbol{w}^{T}(k) \digamma(\boldsymbol{w}(k))} \rightarrow \tau_{c 2}$.

This method provides a numerical method to find the exact value of die-out threshold. In our numerical simulations in Figure 12, we use update law (3.17) to compute the exact values of the die-out threshold.

\subsection{Optimal Information Dissemination}

It is critical to properly design the information dissemination network $B$ to increase $\tau_{c 2}$, thus maximizing the region for which epidemics will die out. For this end, we define a measure of information dissemination impact as:

Definition 2: We define the term "information dissemination robustness gain"

$$
G_{p}=\frac{\tau_{c 2}(\mu)-\tau_{c 1}}{\tau_{c 2}(0)-\tau_{c 1}}
$$

where $\tau_{c 2}(0)$ is the die-out threshold when there is no alertness due to information propagation $(\mu=0)$.

Using (3.6), we derive the approximate analytical formula for $G_{p}$ :

$$
G_{p} \simeq 1+\frac{\mu}{\kappa} \phi_{A}(B)
$$

The information dissemination robustness gain provides us with a measure of the benefit obtained by the use of information dissemination. The greater $G_{p}$ is, the larger the range of effective infection rates for which the epidemic will eventually die out.

The information dissemination index can be maximized by properly designing the information dissemination $B$. If no constraints are set on the maximum number of links that network $B$ can have, then the maximum value of $\phi$ is obtained when $B$ is fully connected. When considering the number of nodes that can broadcast their infection state is constrained to a given value $N^{*}$, the optimal solution is to select the $N^{*}$ nodes that have the largest eigenvector centrality (nodes with largest $v_{1 i}$ ). Finally, when the number of the links $L$ in network $B$ is given, the optimal topology of the information dissemination network is such that $b_{i j}=1$ for the first $L$ pairs $(i, j)$ with highest value of $v_{1 i} v_{1 j}$ and $b_{i j}=0$ for the rest (see [19] for proofs and additional details).

\subsection{Numerical Simulations}

In [22], we constructed a network model of a rural community in Kansas, USA by collecting data on the actual contact patterns and computing rates of contact among a sampled population. The estimated contact network is a weighted graph consisting of 138 nodes and 9222 links with average node strength 0.863 and clustering coefficient $3.7 \times 10^{-3}$. Using this contact network, we computed the information dissemination index under different scenarios. Assume that $p$ percent of individuals are willing to share with the whole community if they are infected. Increasing $p$ from $0 \%$ to $100 \%$ by steps of $10 \%$, we obtained the value of the information dissemination index for each case. We considered two scenarios: 1) individuals are selected randomly; 2) individuals are selected at each step to maximize the information dissemination index. Figure 10 shows when using information of only $10 \%$ of the population in the targeted scenario, the information dissemination index is almost equal to the case of $50 \%$ for the random 


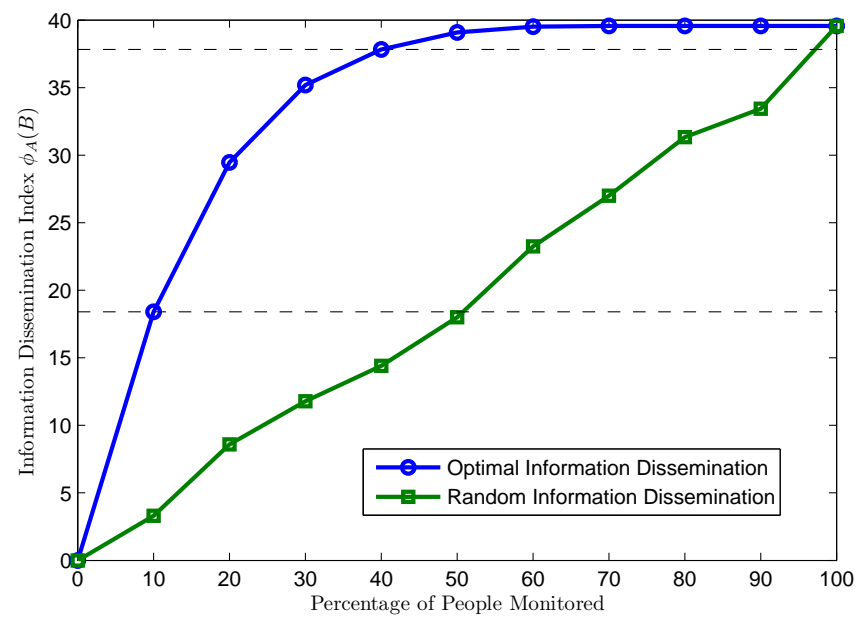

FIGURE 10. Information dissemination index for increasing number of monitored people when the selection is performed randomly (green) and targeted based on the eigenvector centrality (blue).

selection scenario. Furthermore, almost the full potential of information dissemination is achieved using $50 \%$ of the population in the targeted scenario. This example shows the importance of the information dissemination network topology and one possible way to efficiently select the most important information to be shared.

To demonstrate the effect of alertness and information dissemination, we ran extensive simulations to determine the final state of a possible epidemic in the above community. In the classic SIS model, the final size is zero when the effective infection rate $\tau$ is below the no-spread threshold $\tau_{c 1}$, and is positive for $\tau>\tau$ and monotonically increasing by $\tau$. A qualitatively similar behavior is observed for SAIS model, however the threshold point in increased to the greater value $\tau_{c 2}$ (see red curve in Figure 11). In other words, the preventive behavior of alert population widens the range of effective infection rates $(\tau)$ for which the final epidemic state is zero. This die-out region is further expanded for SAIS-ID model because information sharing in information dissemination network promotes more alert behavior (see black curve in Figure 11). The increment of $\tau_{c 2}$ due to the information dissemination depends on the its network structure and can be maximized by properly constructing the network topology, as discussed in the previous section.

Exact values of the die-out threshold in Figure 12 exhibit increment by percentage of monitored people similar to Figure 10, highlighting the importance of information dissemination index. Finally, we compute the robustness gain $G_{p}(3.20)$ as a function of the information dissemination index (Figure 13). As can be observed, the robustness gain increases almost linearly with the information dissemination index, as predicted by the approximate analytical expression for $\tau_{c 2}$ in (3.6). An increase in the die-out threshold is a positive and desirable feature, since the population cannot be widely invaded for a larger range of effective infection rates.

\section{Conclusion}

Inclusion of prophylactic human responses to our epidemic model determines a cushion region for effective infection rates greater than the no-spreading threshold, where the epidemic can still be contained by alerted and preventive human behavior. This cushion region can be further increased by information dissemination on the state of infection of individuals through the use of information networks, typically, on-line social networks. From a network science perspective, we showed maximal efficacy of information dissemination is obtained when the individuals who share their infection state are the ones with greater 


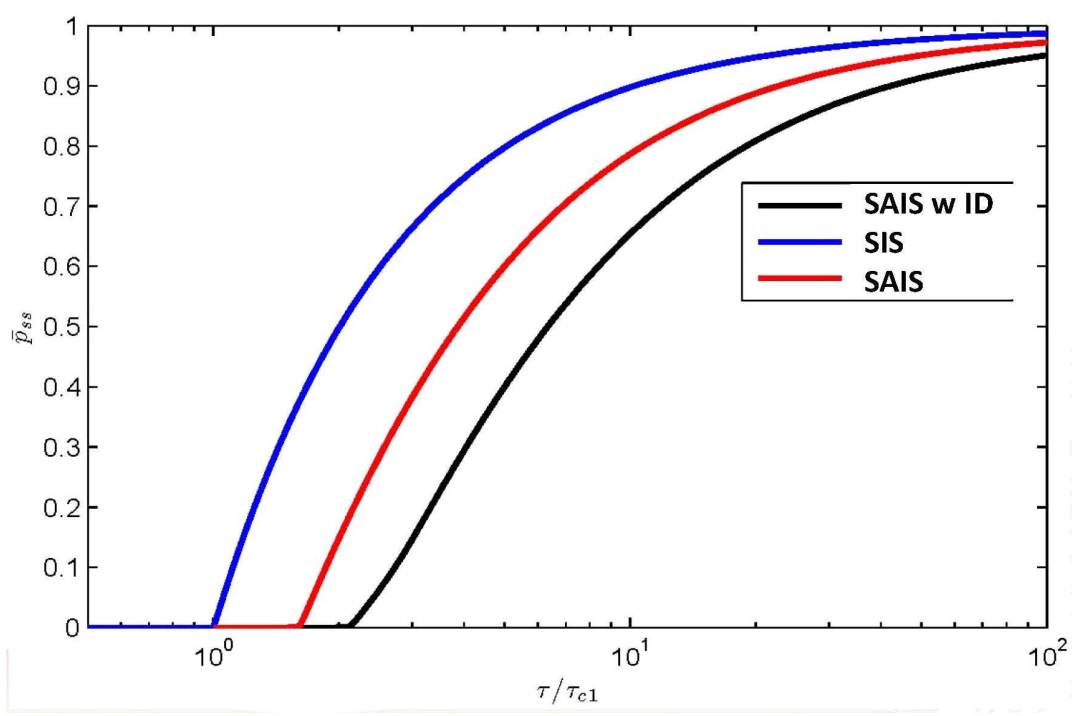

Figure 11. Final infection fractions in the SIS model (blue), the SAIS model (red) and the SAIS model with information dissemination (black). Information dissemination expands the region for effective infection rate $\tau=\beta / \delta$ for which initial infections die-out.

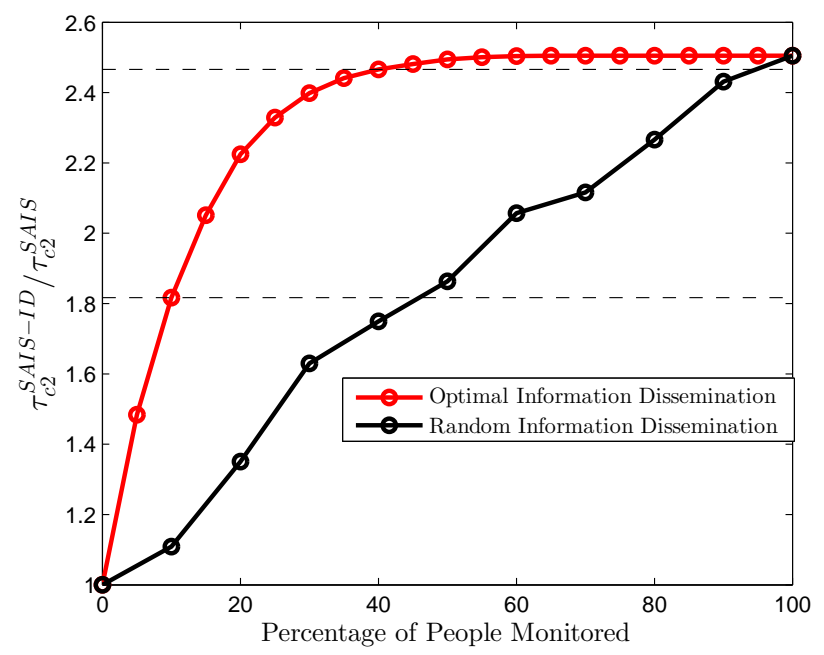

Figure 12. Magnitude of increase of the die-out threshold in SAIS-ID model w.r.t. the SAIS model in case of targeted (red line) versus random (black line) information dissemination.

spectral centrality. Many questions remain open for the eventual practical usability of these results, including critical issues of privacy and the reliability/accuracy of actual behavioral modeling. Despite those limitations, this paper presents sophisticated analytical results that can serve as building blocks for intervention design of real-time epidemic control.

Application to realistic spreading scenarios requires much more complex modeling both at the individual level dynamics and the network topology. Future research is required to incorporate several influential processes in the model, e.g., propagation of alertness, alertness of infected individuals, and influence of symptomatic and asymptomatic compartments. 


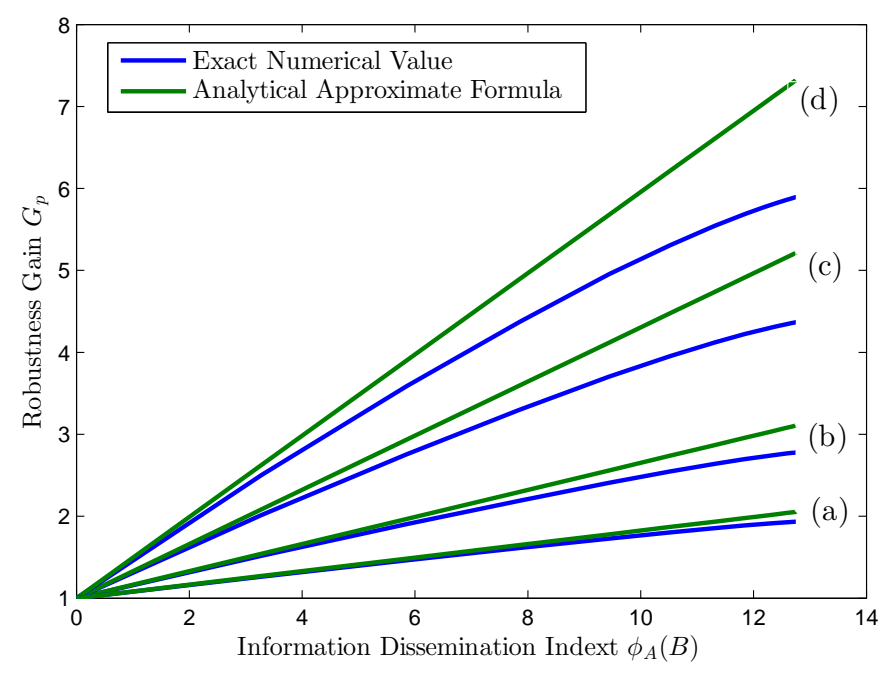

FIGURE 13. Robustness gain as a function of information dissemination index, as shown through exact numerical solution (blue curves) and analytical approximate formula (green lines). For (a) $\mu / \kappa=0.5$, (b) $\mu / \kappa=1$, (c) $\mu / \kappa=2$, (d) $\mu / \kappa=3$.

Acknowledgements. This work was partially supported by the U.S. National Science Foundation, while one of the authors, Fahmida N. Chowdhury, was working at the Foundation. Any opinions, findings, and conclusions or recommendations expressed in this material are those of the authors and do not necessarily reflect the views of the National Science Foundation.

\section{References}

[1] S. Cousens, B. Kanki, S. Toure, I. Diallo, V. Curtis. Reactivity and repeatability of hygiene behaviour: structured observations from burkina faso. Soc. Sci. Med., 43 (1996), No. 9, 1299-1308.

[2] S. Del Valle, H. Hethcote, J. Hyman, C. Castillo-Chavez. Effects of behavioral changes in a smallpox attack model. Math. Biosci., 195 (2005), No. 2, 228-251.

[3] E. P. Fenichel, C. Castillo-Chavez, M. Ceddia, G. Chowell, P. A. G. Parra, G. J. Hickling, G. Holloway, R. Horan, B. Morin, C. Perrings, et al. Adaptive human behavior in epidemiological models. PNAS, 108 (2011), No. 15, 6306-6311.

[4] N. Ferguson. Capturing human behaviour. Nature, 446 (2007), No. 7137, 733.

[5] S. Funk, M. Salath, V. A. A. Jansen. Modelling the influence of human behaviour on the spread of infectious diseases: a review. J. R. Soc. Interface, 7 (2010), 1247-1256.

[6] S. Funk, E. Gilad, C. Watkins, V. Jansen. The spread of awareness and its impact on epidemic outbreaks. PNAS, 106 (2009), No. 16, 6872-6877.

[7] W. R. Gilks, S. Richardson, D. J. Spiegelhalter. Markov chain Monte Carlo in practice. Vol. 2, CRC press, 1996.

[8] O. Givan, N. Schwartz, A. Cygelberg, L. Stone. Predicting epidemic thresholds on complex networks: Limitations of mean-field approaches. J. Theor. Biol., 288 (2011), 21-28.

[9] M. J. Keeling, P. Rohani. Modeling infectious diseases in humans and animals. Princeton Univ. Press, 2008.

[10] B. Kosko. Neural networks and fuzzy systems: a dynamical systems approach to machine intelligence. Prentice-Hall, Inc., 1991.

[11] B. Lemmens, R. Nussbaum. Nonlinear Perron-Frobenius Theory. Vol. 189, Cambridge University Press, 2012.

[12] L. Machado, N. Wyatt, A. Devine, B. Knight. Action planning in the presence of distracting stimuli: An investigation into the time course of distractor effects. J. Exp. Psychol. Hum. Percept. Perform., 33 (2007), No. 5, 1045.

[13] S. Miller, L. Yardley, P. Little. Development of an intervention to reduce transmission of respiratory infections and pandemic flu: Measuring and predicting hand-washing intentions. Psych., Health Med., 17 (2012), No. 1, 59-81.

[14] N. Perra, D. Balcan, B. Gonasalves, A. Vespignani. Towards a characterization of behavior-disease models. PLoS ONE, 6 (2011), No. 8, e23084.

[15] P. Poletti. Human behavior in epidemic modelling, Ph.D. thesis, University of Trento, 2010.

[16] T. Reluga. Game theory of social distancing in response to an epidemic. PLoS Comput. Biol., 6 (2010), No. 5 , e1000793.

[17] F. D. Sahneh, C. Scoglio. Epidemic spread in human networks. in: IEEE Decis. Contr. P., (2011), 3008-3013.

[18] F. D. Sahneh, F. N. Chowdhury, C. M. Scoglio. On the existence of a threshold for preventive behavioral responses to suppress epidemic spreading. Sci. Rep., 2 (2012), 632. 
[19] F. D. Sahneh, C. Scoglio. Optimal information dissemination in epidemic networks. in: IEEE Decis. Contr. P., (2012), 1657-1662.

[20] F. D. Sahneh, C. Scoglio, P. Van Mieghem. Generalized epidemic mean-field model for spreading processes over multilayer complex networks. IEEE/ACM Trans. Networking, 21 (2013), No. 5, 1609-1620.

[21] R. Sapolsky. Why Zebras Dont Get Ulcers. An Updated Guide to Stress, Stress-Related Diseases and Coping. New York: WH Freeman and Company, 1998.

[22] C. Scoglio, W. Schumm, P. Schumm, T. Easton, S. R. Chowdhury, A. Sydney, M. Youssef. Efficient mitigation strategies for epidemics in rural regions. PLoS ONE, 5 (2010), No. 7, e11569.

[23] M. Taylor, P. L. Simon, D. M. Green, T. House, I. Z. Kiss. From markovian to pairwise epidemic models and the performance of moment closure approximations. J. Math. Biol., 64 (2012), No. 6, 1021-1042.

[24] S. M. Tracht, S. Y. Del Valle, J. M. Hyman. Mathematical modeling of the effectiveness of facemasks in reducing the spread of novel influenza A (H1N1). PLoS ONE, 5 (2010), No. 2, e9018.

[25] M. Youssef, C. Scoglio. Mitigation of epidemics in contact networks through optimal contact adaptation. Math. Biosci. Eng., 10 (2013), No. 4, 1227-1251. 